\title{
M étodos de avaliação da composição corporal em pacientes submetidos à hemodiálise
}

\section{M ethods of body composition assessment in patients undergoing hemodialysis}

\author{
Maria Ayako KAM IMURA ${ }^{1}$ \\ Sérgio Antônio DRAIBE² \\ Dirce Maria SIGULEM ${ }^{3}$ \\ Lílian CUPPARI²
}

RE S U M O

A avaliação da composição corporal de pacientes com insuficiência renal crônica em hemodiálise é de suma importância para propiciar uma conduta clínica e nutricional adequada, de forma a contribuir para a redução da elevada taxa de morbidade e mortalidade observada nesta população. No entanto, fatores relacionados à doença tais como anormalidades no estado de hidratação e presença de osteodistrofia renal, podem afetar a validade das técnicas de avaliação da composição corporal de pacientes com insuficiência renal crônica. Apesar dos inúmeros métodos de análise de composição corporal existentes, a maioria apresenta limitações para utilização na prática clínica da população em hemodiálise. Este artigo tem como objetivo revisar os métodos de composição corporal de fácil aplicabilidade na rotina clínica dos pacientes em hemodiálise tais como a somatória de pregas cutâneas, a bioimpedância elétrica e a interactância do infravermelho próximo.

Termos de indexação: composição corporal, hemodiálise, somatória de dobras cutâneas, bioimpedância elétrica, interactância ao infravermelho próximo.

A B S T R A C T

The evaluation of body composition in chronic renal failure patients on hemodialysis is of paramount importance for an adequate clinical and nutritional intervention, so that it can contribute to reduce the high morbidity and

\footnotetext{
${ }^{1}$ Programa de Pós-Graduação em Nutrição, Universidade Federal de São Paulo, Escola Paulista de Medicina. R. Pedro de Toledo, 282, Vila Clementino, 04039-000, São Paulo, SP, Brasil. Correspondência para/Correspondence to: M.A. KAMIMURA. E-mail: m.kamimura@uol.com.br

2 Disciplina de N efrologia, Universidade Federal de São Paulo, Escola Paulista de Medicina, Brasil.

${ }^{3}$ Departamento de Pediatria; Programa de Pós-Graduação em Nutrição, Universidade Federal de São Paulo, Escola Paulista de Medicina, Brasil.
} 
mortality observed in this population. However, factors related to the disease process, such as abnormalities in the hydration status and presence of renal osteodystrophy, may affect the validity of body composition evaluation techniques in patients with chronic renal failure. Although there are a number of studies concerning body composition analysis methods, the majority presents limitations for utilization in clinical practice of the hemodialysis population. This paper aims to review the methods of body composition analysis that are easily applicable in the clinical routine of hemodialysis patients, such as the sum of skinfold thicknesses, bioelectrical impedance analysis and near-infrared interactance.

Index terms: body composition, hemodialysis, skinfold thicknesses, bioelectrical impedance analysis, near-infrared interactance.

\section{N T R O D U Ç Ã O}

A desnutrição energético-protéica em pacientes com insuficiência renal crônica submetidos à hemodiálise tem sido amplamente estudada devido à sua elevada prevalência e estreita associação com a morbidade e mortalidade ${ }^{1}$. Por outro lado, evidências de sobrepeso e obesidade nesta população vêm sendo relatadas mais recentemente. Alguns estudos sugerem que o excesso de peso, considerado um fator de risco para mortalidade em indivíduos saudáveis, apresenta uma influência positiva na sobrevida dos pacientes em hemodiálise ${ }^{2}$. Frente a tais condições, a identificação dos diferentes compartimentos corporais, incluindo os estoques de proteína e gordura corporais na avaliação nutricional, é de fundamental importância na rotina clínica de pacientes submetidos à hemodiálise crônica.

0 processo de hemodiálise pode levar à remoção de 1 a 4 litros de fluido no período médio de 4 horas e, dependendo do paciente e da eficiência da diálise, as alterações no volume do fluido corporal podem resultar em situações que variam desde edema e congestão pulmonar até hipotensão e desidratação ${ }^{3}$. Estas constantes variações hídricas podem tornar imprecisas as medidas da composição corporal, dificultando a avaliação e o acompanhamento do estado nutricional ${ }^{4,5}$.

A pesar das inúmeras técnicas de aferição dos compartimentos corporais existentes, a maioria apresenta limitações na sua aplicação. A identificação de uma técnica simples, de baixo custo, que não ofereça riscos e que possa medir adequadamente os compartimentos corporais seria de grande utilidade para o acompanhamento nutricional de pacientes em hemodiálise. Sendo assim, o presente artigo tem por objetivo revisar a aplicabilidade dos métodos simples de avaliação da composição corporal tais como a somatória de pregas cutâneas, a bioimpedância elétrica e a interactância do infravermelho em pacientes submetidos à hemodiálise crônica.

\section{COM POSIÇÃ O CORPORAL DE PACIENTES EM HE M O DIÁ LISE}

0 estado nutricional do paciente com insuficiência renal crônica é afetado por diversas condições como a anorexia, presença de toxinas urêmicas, distúrbios gastrintestinais e alterações metabólicas ${ }^{6}$. Além disso, nos pacientes submetidos ao tratamento de hemodiálise, os estímulos catabólicos do procedimento dialítico per se, a perda de nutrientes para o dialisato e a acidose metabólica são fatores que também contribuem para 0 estado de desnutrição ${ }^{7}$, podendo levar a alterações na composição corporal. De fato, os estudos mostram que os homens em hemodiálise tendem a apresentar reduzida massa muscular e as mulheres um menor estoque de gordura corporal, quando comparados aos parâmetros antropométricos de indivíduos saudáveis ${ }^{8}$. Poucos estudos avaliaram prospectivamente as modificações das reservas protéicas 
e de gordura corporal ao longo da terapia crônica de hemodiálise utilizando métodos de alta precisão e reprodutibilidade.

Recentemente, alguns estudos realizados com a técnica da absortometria de Raios $X$ de dupla energia, têm demonstrado que, no primeiro ano de tratamento hemodialítico, ocorre uma redução da massa magra corporal ${ }^{9}$. Visto que a massa magra é um compartimento que varia de acordo com o estado de hidratação, os autores atribuíram essa diminuição da massa magra como um reflexo da remoção gradual do fluido corporal para atingir o peso seco durante o período inicial de tratamento. Por outro lado, tem sido evidenciado, principalmente nos pacientes desnutridos, um aumento significante de gordura corporal no primeiro ano de hemodiálise ${ }^{9,10}$. Esta condição pode ocorrer mais provavelmente como resultado da redução de toxinas urêmicas circulantes e conseqüente melhora do apetite.

A manutenção de uma reserva adequada de gordura corporal é vantajosa para o paciente em hemodiálise, pois em situações de maior necessidade energética como nas infecções, nas cirurgias repetidas do acesso vascular ou no transplante renal, a gordura armazenada pode ser utilizada para suprir o déficit energético e poupar a utilização das reservas protéicas.

\section{Avaliação da composição corporal}

O modelo de dois compartimentos (massa magra e gordura corporal), na qual se baseiam muitos dos métodos de avaliação da composição corporal, assume que, assim como na gordura corporal, todos os elementos que compõem a massa magra (predominantemente água, proteínas e minerais) encontram-se nas mesmas proporções para todos os indivíduos. Este último é particularmente improvável nos pacientes com insuficiência renal crônica, que apresentam como principal conseqüência da doença, alterações hidroeletrolíticas ${ }^{11}$. Além disso, a presença de osteodistrofia renal com perda da massa óssea, condição não raramente encontrada nesta população, pode representar outra fonte de erro na avaliação dos compartimentos corporais ${ }^{12}$. De fato, Woodrow et al. ${ }^{12}$ demonstraram que a magnitude de erro das técnicas de composição corporal era mais acentuada em grupos de pacientes renais crônicos que em indivíduos saudáveis

Os inúmeros estudos de avaliação da composição corporal em pacientes com insuficiência renal crônica mostram uma relativa variabilidade entre os diferentes métodos na avaliação dos compartimentos corporais. Este fato é esperado, mesmo na população de indivíduos saudáveis, em razão dos diferentes princípios em que se baseiam cada uma das técnicas e das equações de predição utilizadas para avaliar um componente corporal in vivo. Além disso, cada método apresenta diferentes níveis de precisão, acurácia, sensibilidade e reprodutibilidade, podendo comportar-se diferentemente, dependendo da população e situações clínicas específicas.

A absortometria de Raios X de dupla energia que foi inicialmente desenvolvida para avaliar o conteúdo mineral ósseo na investigação de doenças como a osteoporose, é considerada, atualmente, um método de boa precisão e reprodutibilidade para avaliar a composição corporal ${ }^{13}$. Esta técnica é baseada na atenuação de raios em diferentes níveis de energia e permite realizar a mensuração corporal total e por segmentos (cabeça, tronco e membros).

O princípio básico da absortometria é a utilização de uma fonte de Raios $X$ com um filtro que converte um feixe de Raios $X$ em picos fotoelétricos de baixa e alta energia que atravessam o corpo do indivíduo. A obtenção dos compartimentos corporais é feita pela medida da atenuação desses picos fotoelétricos. A principal limitação deste método está associada à incapacidade de detectar a quantidade de água contida na massa magra. Isto se deve ao fato de que os principais componentes da massa magra, as proteínas e os fluidos corporais, apresentam 
coeficientes de atenuação similares, de tal forma que não são diferenciadas pela absortometria. Sendo assim, o método da absortometria de Raios $X$ de dupla energia estima a água corporal total, assumindo que a mesma é equivalente à uma fração fixa de $73,2 \%$ da massa magra do indivíduo.

Erros sistemáticos poderiam ser esperados, já que a água do corpo pode variar em determinadas condições clínicas como na insuficiência renal crônica. No entanto, os estudos realizados com pacientes em hemodiálise mostram que as modificações na água corporal são detectadas pela absortometria de Raios $X$ de dupla energia por meio das variações ocorridas na massa magra corporal. Abrahamsen et al. ${ }^{14}$ avaliaram pacientes em hemodiálise nos momentos pré e pós a sessão de diálise e encontraram uma forte correlação das medidas de massa magra obtidas pela absortometria de Raios $X$ de dupla energia com os valores de água corporal obtidos pela variação de peso corporal $(r=0.975, p<0.0001)$.

Apesar da necessidade de mais estudos de validação para ser aceito como método padrão ouro na avaliação da composição corporal, a absortometria de Raios $X$ de dupla energia vem sendo utilizada como método de referência em vários estudos comparativos de composição corporal devido a sua elevada precisão, sendo o método recomendado pelo National Kidney Foundation ${ }^{15}$.

A absortometria de Raios $X$ de dupla energia é de rápida execução, não invasiva e não necessita de nenhum preparo ou requisito especial para a execução do exame, no entanto, o método requer um local adequado, equipamento sofisticado, um avaliador treinado e apresenta custo elevado. Dessa forma, esta técnica, assim como as outras mais complexas como a tomografia computadorizada, ressonância magnética, hidrodensitometria, diluição de isótopos e contagem de potássio corporal, ficam limitadas para utilização na rotina clínica do paciente renal crônico.

\section{SOM ATÓ RIA DE PRE G A S C U TÂ N E A S, B IO I M PE D Â NCIA ELÉTRICA E I N TERACT Â NCIA DO I N FRA VER M E L H O PRÓ XIMO}

Dentre as diversas técnicas de aferição dos compartimentos corporais disponíveis na rotina clínica dos serviços de hemodiálise, o tradicional método da somatória de pregas cutâneas e 0 método da bioimpedância elétrica têm sido adotados com maior freqüência em função da praticidade, rapidez e pelo custo relativamente baixo.

$\mathrm{Na}$ técnica da somatória de pregas cutâneas, a composição corporal é estimada utilizando-se a somatória das pregas bicipital, tricipital, subescapular e supra-ilíaca, segundo a equação de Durnin e Womersley: densidade corporal $=(A-B) \cdot \log \Sigma 4$ pregas, onde $A$ e B são coeficientes elaborados de acordo com a idade e o gênero. A partir do valor de densidade corporal, a porcentagem de gordura corporal total é determinada utilizando a fórmula de Siri: gordura corporal $(\%)=4,95 /$ densidade corporal-4,50.100.

A massa corporal magra é obtida subtraindo-se a gordura corporal do peso total do indivíduo. A pesar de alguns autores referirem que as medidas dos compartimentos corporais obtidas pela somatória de pregas cutâneas podem ser influenciadas por alterações hídricas de pacientes portadores de insuficiência renal crônica ${ }^{14}$, o seu uso é favorecido por outros estudiosos ${ }^{16}$.

A bioimpedância elétrica é um método rápido e não-invasivo para estimar os compartimentos corporais, inclusive a distribuição dos fluidos corporais nos espaços intra e extracelulares ${ }^{3}$. A bioimpedância elétrica baseia-se no princípio de que os componentes corporais oferecem uma resistência diferenciada à passagem da corrente elétrica. Os tecidos magros são altamente condutores de corrente elétrica devido a grande quantidade de água e eletrólitos, 
ou seja, apresentam baixa resistência à passagem da corrente elétrica. Por outro lado, a gordura, o osso e a pele constituem um meio de baixa condutividade apresentando, portanto, elevada resistência.

Uma corrente elétrica imperceptível de 500 a $800 \mu \mathrm{A}$ e $50 \mathrm{kHz}$ é introduzida pelos eletrodos distais e captada pelos eletrodos proximais, gerando vetores de resistência (medida de oposição pura ao fluxo de corrente elétrica através do corpo) e reactância (oposição ao fluxo de corrente causada pela capacitância produzida pela membrana celular). Assim, após identificar os níveis de resistência e reactância do organismo à corrente elétrica, o analisador avalia a água corporal total e, assumindo uma hidratação constante, prediz a quantidade de massa magra. Porém, se o indivíduo apresentar hiperhidratação o valor da massa magra fica superestimado ${ }^{17}$. Portanto, a alteração no estado de hidratação é a principal limitação deste método.

O uso da bioimpedância elétrica tem demonstrado eficiência na aferição dos compartimentos corporais em diversas situações clínicas como desnutrição, traumas, pré e pós operatório, doenças hepáticas, insuficiência renal, gestação e, em crianças e atletas. De fato, a bio impedância elétrica é um método de avaliação da composição corporal altamente aceito pela comunidade científica. Porém, seus resultados podem ser afetados por fatores como a alimentação, o exercício físico e a ingestão de líquidos em períodos que antecedem a avaliação, estados de desidratação ou retenção hídrica, utilização de diuréticos e ciclo menstrual.

Em pacientes renais crônicos, alguns autores não recomendam a utilização da bioimpedância elétrica na mensuração das variações agudas de fluido corporal de pacientes em diálise e referem que este método seria útil em estudos de seguimento e não comparativos ${ }^{4}$. Contrariamente, outros estudiosos favorecem a sua aplicação na avaliação das mudanças hídricas agudas da diálise ${ }^{12}$. Woodrow et al. ${ }^{12}$ avaliaram a acurácia da bioimpedância elétrica para a medida da água corporal em pacientes com insuficiência renal crônica, utilizando a diluição do óxido deutério como referência. A bioimpedância elétrica mostrou menor precisão no grupo de pacientes renais crônicos que no grupo composto por indivíduos saudáveis. Além disso, os limites de variação foram maiores no grupo de pacientes em hemodiálise e em tratamento conservador. Os autores atribuem à variabilidade anormal na distribuição de água intra e extracelular como a causa principal da reduzida acurácia na mensuração da água corporal total destes pacientes.

Estudos mais recentes têm verificado que os valores de reactância e do ângulo de fase, derivados da bioimpedância elétrica, apresentam boa correlação com os parâmetros nutricionais e com os índices de morbidade de pacientes submetidos à hemodiálise crônica. E ainda, Guida et al. ${ }^{18}$ detectaram reduzidos índices de massa celular e ângulo de fase obtidos pela bioimpedância nos pacientes com sobrepeso e obesidade, sugerindo que os indivíduos submetidos à terapia crônica de hemodiálise podem estar em risco de desnutrição apesar de apresentarem valores de índice de massa corporal acima do limite de normalidade.

É atribuído uma considerável vantagem ao método da bioimpedância elétrica, quando comparada à somatória de pregas cutâneas, devido à capacidade de mensuração da água corporal e por apresentar menor erro intra e interobservador ${ }^{19}$. No entanto, a somatória de pregas cutâneas e a bioimpedância elétrica têm demonstrado resultados similares na avaliação da composição corporal de pacientes em hemodiálise ${ }^{12,20,21}$.

Quando Woodrow et al. ${ }^{12}$ estudaram a validade dos métodos da somatória de pregas cutâneas e da bioimpedância elétrica em pacientes com insuficiência renal crônica utilizando o método da absortometria de Raios $X$ de dupla energia como referência, os autores constataram que ambos os méto dos mostravam valores similares e concordância com as medidas obtidas pela absortometria de Raios X de dupla energia. 
Avesani et al. ${ }^{22}$ e Stall et al. ${ }^{23}$ observaram fortes correlações entre a somatória de pregas cutâneas ou a bioimpedância elétrica e a absortometria de Raios X de dupla energia em pacientes com insuficiência renal crônica.

Poucos estudos conduziram uma análise comparativa de composição corporal separando os indivíduos por gênero. Stall et al. ${ }^{23}$ observaram em pacientes em diálise peritoneal que a gordura corporal medida por diferentes métodos variou significantemente tanto nos homens quanto nas mulheres. Em um recente estudo com pacientes em hemodiálise, os métodos da somatória de pregas cutâneas e a bioimpedância elétrica mostraram resultados similares aos da absortometria de Raios X de dupla energia ${ }^{21}$. No entanto, quando analisados separadamente por gênero, o método da bioimpedância elétrica mostrou uma tendência a subestimar as medidas de gordura corporal nos homens e a superestimar os valores nas mulheres.

A variabilidade pode ser explicada em parte pela diferença na composição corporal entre os gêneros e pelos princípios em que se baseia a técnica. As fórmulas contidas nos softwares do aparelho de bioimpedância elétrica estimam primariamente a água corporal total por meio dos vetores derivados e, assumindo uma constante de hidratação de $73 \%$ da massa livre de gordura, obtêm a gordura corporal. Assim, estas equações de predição resultam em uma maior margem de erro na mensuração da gordura corporal em virtude da dependência de duas medidas anteriores. E o erro pode encontrar-se mais acentuado em situações de alterações no estado hídrico que ocorre nos pacientes com insuficiência renal crônica. De fato, Woodrow et al. ${ }^{12}$ mostraram que a variabilidade entre os métodos da bioimpedância elétrica e da absortometria de Raios X de dupla energia eram maiores para a mensuração da gordura corporal que da massa livre de gordura. A somatória de pregas cutâneas mostrou-se similar à absorto-metria de Raios X de dupla energia na mensuração da gordura corporal mesmo quando analisada separadamente por gênero ${ }^{21}$. Este último estudo sugere que a somatória de pregas cutâneas apresenta resultados mais precisos em relação à bioimpedância elétrica na avaliação da gordura corporal de pacientes em hemodiálise e os autores enfatizam a importância de se conduzir um estudo comparativo de composição corporal por gênero.

Por fim, dentre os métodos simples de composição corporal atualmente estudados na insuficiência renal crônica, a técnica relativamente recente da interactância do infravermelho próximo vem despertando interesse por ser uma técnica simples, não-invasiva e de fácil execução ${ }^{24}$. Resultados preliminares de uma pesquisa realizada pelo Departamento de Agricultura dos Estados Unidos na análise de alimentos através da espectroscopia do infravermelho, levaram ao desenvolvimento desta técnica na avaliação da composição corporal em humanos.

A interactância do infravermelho próximo baseia-se nos princípios de absorção e reflexão do raio infravermelho para avaliar indiretamente a quantidade de gordura e água corporais. 0 analisador usualmente utilizado é o Futrex ${ }^{\circledR}$, aparelho portátil, composto por um mini-computador, um protetor de luz e um sensor que é apoiado sob o bíceps para a emissão da luz. A luz infravermelha penetra nos tecidos em uma profundidade de até $4 \mathrm{~cm}$ e é refletida pelo osso de volta ao detector, sendo que os comprimentos de onda de $930 \mathrm{~nm}$ e $970 \mathrm{~nm}$ têm sido identificados como os picos de absorção da luz para a gordura e água, respectivamente ${ }^{24}$.

Apesar deste método ter sido criticado pelo fato de levar em consideração apenas um ponto do corpo, na avaliação de várias áreas anatômicas, o bíceps mostrou a melhor correlação com o método de referência da hidrodensitometria ${ }^{25}$. Entretanto, todos os estudos comparativos realizados com o método do infravermelho próximo têm demonstrado uma subestimação da gordura corporal em relação a outras técnicas de composição corporal. Elia et al. ${ }^{25}$ avaliaram indivíduos saudáveis utilizando como método padrão a hidrodensitometria e, de fato, a interactância do infravermelho próximo mostrou 
uma tendência à subestimação das medidas de gordura corporal, especialmente em indivíduos com maior reserva de tecido adiposo. Quando os autores avaliaram separadamente um grupo de mulheres com IM C $>50 \mathrm{~kg} / \mathrm{m}^{2}$, a subestimação chegava a $16 \%$.

Outros pesquisadores relataram a subestimação dos valores de gordura em indivíduos obesos e, ainda, uma superestimação da gordura corporal em indivíduos magros ${ }^{26}$. Sugere-se que a penetração profunda do feixe de luz pode ser rompida pelas camadas e irregularidades da junção gordura-músculo, principalmente em indivíduos com maior reserva de tecido adiposo.

Em pacientes submetidos à hemodiálise, os resultados obtidos pela interactância do infravermelho próximo têm mostrado uma boa correlação com a avaliação global subjetiva, medidas antropométricas, assim como com os parâmetros laboratoriais nutricionalmente relevantes tais como a albumina, a transferrina, o colesterol e a taxa de redução de uréia ${ }^{27}$. Em um recente estudo longitudinal, Kalantar-Zadeh et al. ${ }^{27}$ acompanharam 71 pacientes durante o período de um ano e concluíram que a interactância do infravermelho próximo apresenta boa reprodutibilidade e é um instrumento útil para avaliar as modificações de gordura corporal ao longo do tempo em pacientes submetidos à diálise ${ }^{28}$. No entanto, os estudos citados anteriormente não utilizaram outros métodos de composição corporal para análise comparativa.

A té o momento, apenas dois estudos de composição corporal em pacientes renais crônicos incluíram o método da interactância do infravermelho próximo para comparação. Em 1994, Lo et al. ${ }^{29}$ avaliaram a massa magra de pacientes em diálise peritoneal utilizando diferentes métodos de composição corporal, e os resultados obtidos pela interactância do infravermelho próximo mostraram-se significantemente maiores em relação à massa magra medida pelo método da contagem total de potássio utilizado como referência no estudo. Em uma recente pesquisa incluindo 90 pacientes em hemodiálise, o método da interactância do infravermelho próximo foi comparado à somatória de pregas cutâneas e à bioimpedância elétrica para a determinação da gordura corporal ${ }^{30}$. Neste único estudo em que o método do infravermelho próximo foi analisado na população em hemodiálise, os achados denotam uma subestimação das medidas de gordura corporal, confirmando os resultados obtidos em estudos com indivíduos saudáveis.

Condições como a capacidade de penetração do raio infravermelho, as características da derme e a quantidade de água corporal têm sido citados como fatores que podem afetar os resultados da interactância do infravermelho próximo.

\section{CONSIDERACÕ ES FINA IS}

Estudos enfocando a desnutrição energético-protéica e as situações de sobrepeso e obesidade em pacientes submetidos à hemodiálise são de extrema relevância visto a sua influência sobre a taxa de morbidade e mortalidade nesta população. A avaliação da composição corporal é imprescindível para a detecção e prevenção de tais condições e para a elucidação das conseqüências metabólicas decorrentes da enfermidade e do tratamento de hemodiálise crônica. Para tanto, são fundamentais a escolha de uma técnica de composição corporal adequada, o conhecimento dos princípios e das equações de predição do instrumento e uma aplicação cuidadosa. Além disso, a avaliação periódica dos compartimentos corporais dos pacientes faz-se necessária para o acompanhamento das modificações da composição corporal ao longo da terapia dialítica.

A pesar das divergências dos resultados de análise da composição corporal nos grupos de pacientes com insuficiência renal crônica, esta revisão mostra que, dentre os métodos simples de avaliação da composição corporal de pacientes 
em hemodiálise, o tradicional método da somatória de pregas cutâneas parece apresentar uma vantagem visto os resultados confiáveis, além da praticidade e o baixo custo. Porém, há necessidade de mais estudos de acompanhamento que avaliem a reprodutibilidade desta técnica na população em hemodiálise.

\section{REFERÊ N C I A S}

1. Qureshi AR, Alvestrand A, Divino-Filho JC, Gutierrez A, Heimburger O, Lindholm B, et al. Inflammation, malnutrition, and cardiac disease as predictors of mortality in hemodialysis patients. J Am Soc Nephrol 2002; 13:S28-S36.

2. Kopple JD, Zhu X, Lew NL, Lowrie EG. Body weightfor-height relationships predict mortality in maintenance hemodialysis patients. Kidney Int 1999; 56:1136-48.

3. Kushner RF, De Vries PMJP, Gudivaka R. Use of bioelectrical impedance analysis measurements in the clinical management of patients undergoing dialysis. Am J Clin Nutr 1996; 64:S503-S9.

4. Kurtin PS, Shapiro AC, Tomita H, Raizman D. Volume status and body composition of chronic dialysis patients: utility of bioelectric impedance plethysmography. Am J Nephrol 1990; 10:363-7.

5. Kamimura M A, Schumaker F, Agliussi RG, Baxmann A, Canziani MEF, Cuppari L, et al. Effect of body water changes on body composition assessed by near-infrared compared to bioelectrical impedance and skinfold thicknesses in hemodialysis patients". Annals of the 10th International Congress on Nutrition and Metabolism in Renal Disease; 2000; Lyon, France. France: International Society of Renal Nutrition \& M etabolism; 2000. p.49.

6. Kaufmann P, Smolle KH, Horina JH, Zach R, Krejs GJ. Impact of long-term hemodialysis on nutritional status in patients with end-stage renal failure. Clin Investig 1994; 72:754-61.

7. Ikizler TA, Pupim LB, Brouillette JR, Levenhagen DK, Farmer K, Hakim RM, et al. Hemodialysis stimulates muscle and whole body protein loss and alters substrate oxidation. Am J Physiol Endocrinol Metab 2002; 282:107-16.

8. Cuppari L, Draibe AS, Anção MS, Sigulem D, Sustovich DR, Ajzen $\mathrm{H}$, et al. Avaliação nutricional em pacientes renais crônicos em programa de hemodiálise. Estudo multicêntrico. Assoc Med Bras 1989; 35.

9. Ishimura E, Okuno S, Kim M, Yamamoto T, Izumotani T, Otoshi T, et al. Increasing body fat mass in the first year of hemodialysis. J Am Soc Nephrol 2001; 12:1921-6.

10. Pupim LB, Kent P, Caglar K, Shyr Yu, Hakim RM, Ikizler TA. Improvement in nutritional parameters after initiation of chronic hemodialysis. Am J Kidney Dis 2002; 40:143-51.

11. Brennan BL, Yasumura S, Letteri JM , Cohn SH. Total body electrolyte composition and distribution of body water in uremia. Kidney Int 1980; 17: 364-71.

12. Woodrow G, Oldroyd B, Smith MA, Turney JH. $M$ easurement of body composition in chronic renal failure: comparison of skinfold anthropometry and bioelectrical impedance with dual energy $X$-ray absortiometry. Eur J Clin Nutr 1996; 50:295-301.

13. Mazess RB, Barden HS, Bisek JP, Hanson J. Dual-energy $\mathrm{X}$-ray absorptiometry for total body and regional bone mineral and soft-tissue composition. Am J Clin Nutr 1990; 51:1106-12.

14. A brahamsen B, Hansen TB, Hogsberg IM , Pedersen $\mathrm{FB}$, Beck-Nielsen $\mathrm{H}$. Impact of hemodialysis on dual $\mathrm{X}$-ray absorptiometry, bioelectrical impedance mesurements, and antropometry. Am J Clin Nutr 1996; 63:80-6.

15. National Kidney Foundation: DOQI Kidney Disease Outcomes Quality Initiative Clinical Practice Guidelines for Nutrition in Chronic Renal Failure. Am J Kidney Dis 2000; 35(Suppl 2):34-5.

16. Nelson EE. Anthropometry in the nutritional assessment of adults with end-stage renal disease. J Renal Nutr 1991; 1(4):162-72.

17. De Lorenzo A, Barra PFA, Sasso GF, Battistini NC, Deureberg P. Body impedance measurements during dialysis. Eur J Clin Nutr 1991; 45:321-5. 
18. Guida B, De Nicola L, Pecoraro P, Trio R, Di Paola F, lodice $C$, et al. Abnormalities of bioimpedance measures in overweight and obese hemodialyzed patients. Int J Obes 2001; 25:265-72.

19. Lukaski HC, Bolonchuk WW, Hall CB, Siders WA. Validation of tretapolar bioelectrical impedance method to assess human body composition. J Appl Physiol 1986; 60:1327-32.

20. Oe B, De Fijter CWH, Oe PL, Stevens P, De Vries PMJM. Four-sites skinfold anthropometry (FSA) versus body impedance analysis (BIA) in assessing nutritional status of patients on maintenance hemodialysis: which method is to be preferred in routine patient care? Clin Nephrol 1998; 49(3):180-5.

21. Kamimura MA, Avesani CM, Cendoroglo M, Canziani MEF, Draibe SA, Cuppari L. Comparison of skinfold thicknesses and bioelectrical impedance analysis with dual-energy $X$-ray absorptiometry for the assessment of body fat in patients on long-term hemodialysis therapy. Nephrol Dial Transplant 2003; 18(1):101-5.

22. Avesani CM , Cuppari L, Ventura RTP, Cendoroglo $M$, Draibe SA. Comparison of body composition assessed by three methods in nondialyzed chronic renal failure patients. Annals of the 10th International Congress on Nutrition and M etabolism in Renal Disease; 2000; Lyon, France. France: International Society of Renal Nutrition \& M etabolism; 2000. p.60.

23. Stall SH, Ginsberg NS, De Vita MV, Zabetakis PM, Lynn Rl, Gleim GW, et al. Comparison of five body-composition methods in peritoneal dialysis patients. Am J Clin Nutr 1996; 64:125-30.
24. Conway JM, Norris KH, Bodwell CE. A new approach for the estimation of body composition: infrared interactance. Am J Clin Nutr 1984; 40:1123-30.

25. Elia M, Parkinson SA, Diaz E. Evaluation of near infra-red interactance as a method for predicting body composition. Eur J Clin Nutr 1990; 44: 113-21.

26. Brooke-Wavell K, Jones PRM, Norgan NG, Hardman AE. Evaluation of near infra-red interactance for assessment of subcutaneous and total body fat. Eur J Clin Nutr 1995; 49:57-65.

27. Kalantar-Zadeh K, Dunne E, Nixon K, Kahn K, Lee $\mathrm{GH}$, Kleiner $M$, et al. Near infra-red interactance for nutritional assessment of dialysis patients. Nephrol Dial Transplant 1999; 14:169-75.

28. Kalantar-Zadeh K, Block G, Kelly M P, Schroepfer C, Rodriguez RA, Humphreys MH. Near Infra-Red Interactance for Longitudinal Assessment of Nutrition in Dialysis Patients. J Renal Nutr 2001; 11:23-31.

29. Lo W, Prowant BF, Moore HL, Gamboa SB, Nolph $K D$, Flynn $M A$, et al. Comparison of different measurements of lean body mass in normal individuals and in chronic peritoneal dialysis patients. Am J Kidney Dis 1994; 23:74-85.

30. Kamimura M A, Santos NSJ, Avesani CM , Canziani MEF, Draibe SA, Cuppari L. Comparison of simple methods for the determination of body fat in patients on long-term hemodialysis therapy. J Am Diet Assoc 2003; 103(2):195-9.

Recebido para publicação em 22 de outubro de 2002 e aceito em 8 de maio de 2003. 
